\title{
Can Digital Therapeutics Open a New Era of Sleep Medicine?
}

\author{
Kyung Mee Park ${ }^{1,2}$, Suonaa Lee², and Eun Lee ${ }^{2}$ \\ 'Department of Hospital Medicine, Yongin Severance Hospital, Yonsei University College of Medicine, Yongin, Korea \\ 2Department of Psychiatry and Institute of Behavioral Science in Medicine, Yonsei University College of Medicine, Seoul, Korea
}

Digital therapeutics (DTx) are products that provide evidence-based interventions driven by high-quality software programs to prevent, manage, or treat a medical disease. DTx are receiving increasing attention as a new therapeutic approach. Several DTx for insomnia are on the market, some of which have received approval by national regulatory agencies. DTx for insomnia are usually based on cognitive behavioral therapy for insomnia. No DTx for other sleep disorders, such as narcolepsy or sleep-related breathing disorders, have received regulatory authority approval as a medical device. DTx have the substantial benefits of being accessible and relatively low-cost. However, several issues related to DTx have not yet been fully resolved, and discussions regarding DTx are still in the early stages. To use DTx for sleep disorders as an effective treatment option in the future, considering the current status of DTx is necessary. This review discusses definitions and background of DTx; specific DTx for insomnia that have been developed; use of DTx for sleep and related psychiatric comorbid symptoms; global regulatory processes for DTx, including prescribing and medical billing issues; and remaining challenges regarding the use of DTx.

Keywords: Digital technology; eHealth; Software; Insomnia; Sleep disorder $\mathrm{nc} / 4.0)$ which permits unrestricted non-commercial use, distribution, and reproduction in any medium, provided the original work is properly cited.

\section{INTRODUCTION}

The field of digital therapeutics (DTx) has grown steadily over the past decade. The academic term "digital therapeutics" was first proposed in 2015 and defined as "evidence-based behavioral treatments delivered online that can increase accessibility and effectiveness of healthcare" [1]. In 2017, an application for managing substance use disorder known as reSET ${ }^{\circledR}$ (Pear Therapeutics) received authorization from the United States (US) Food and Drug Administration (FDA) [2]. Before reSET ${ }^{\circledR}$, other digital healthcare software included Bluestar ${ }^{\circledR}$ (Welldoc Inc.), a type 2 diabetes management mobile application that received FDA authorization in 2010 [3]. Nevertheless, reSET $^{\circledR}$ is considered the earliest FDA-approved DTx, as it was the first for which treatment benefits were claimed at the time of approval, with clinical trial evidence provided to support this claim. Treatment with re$\mathrm{SET}^{\circledR}$ involves a prescription from a physician, similar to traditional medical treatment.

Since the approval of reSET ${ }^{\circledR}$, DTx have received increasing at- tention as a new paradigm for medical treatment. The purpose of this article is to examine the current and future roles of DTx in the field of sleep medicine. We first review definitions and background of DTx and then discuss use of DTx developed for insomnia and other sleep disorders, use of DTx for patients with sleep disorders who have psychiatric comorbidities, and regulations for DTx in various regions of the world. We review studies comparing DTx with existing treatments, describe challenges associated with the use of DTx, and discuss the future of DTx in sleep medicine.

\section{DEFINITION AND BACKGROUND OF DIGITAL THERAPEUTICS}

DTx are defined as products driven by a high-quality software program that provides evidence-based interventions for patients to aid in the prevention, management, or treatment of a medical disorder [4]. Digital health is a concept that can be applied to general healthcare using digital technology [5] that broadly consists of technologies, platforms, and systems that engage subjects to 
promote optimization of their lifestyle, wellness, and health. DTx are a subdivision of digital health. While digital health includes both hardware and software, DTx involve only software. In this regard, DTx can be considered a type of software as a medical device (SaMD), a term referring to software used for one or more medical purposes, without accompanying hardware [6]. The key characteristic that distinguishes DTx from digital health or SaMDs is the purpose of DTx: to prevent, manage, or treat medical diseases [4]. Because DTx are used for therapeutic purposes, they require a higher level of evidence than other digital health products aimed at pure-play adherence, diagnostics, and telehealth [4]. As such, DTx can be expected to have a higher level of clinical effects than general wellness digital health products.

The Digital Therapeutics Alliance (DTA) classifies a DTx into three categories according to its purpose: treatment, disease management, and health promotion [4]. DTx for therapeutic purposes require validation of treatment efficacy and safety by a third party and may result in charges to insurance companies through prescriptions from medical professionals. DTx for health promotion have fewer issues with medical billing, and validation issues related to effectiveness or safety are relatively less important. DTx for promoting health have a lower risk of medical claims than DTx for other purposes.

DTx can also be classified as a digital companion or replacement therapy [7]. DTx as a digital companion are products that provide therapeutic effects through indirect methods that improve convenience and effectiveness by supplementing traditional treatments, such as pharmacologic treatment. This form of DTx is used with traditional, existing treatments. Conversely, DTx as a replacement therapy are products with their own therapeutic benefits. This form of DTx can replace conventional treatment or be used in combination with usual therapy to enhance treatment effects. For any purpose, clinical evidence of therapeutic effects is required for products to be called DTx.

\section{DIGITAL THERAPEUTICS FOR INSOMNIA}

Cognitive behavioral therapy for insomnia (CBTi) is recommended as first-line treatment for insomnia in many treatment guidelines. CBTi is a well-established effective nonpharmacologic therapy that adjusts maladaptive cognition and behaviors related to insomnia [8]. Despite similar or superior treatment effects when compared with pharmacologic therapy [9], CBTi has the disadvantages of lower accessibility and higher costs $[10,11]$. To resolve these limitations, ongoing efforts have focused on providing CBTi through online processes, which have shown significant results [10]. Most DTx for insomnia are based on digital CBTi (dCBTi).

dCBTi is emerging as an ideal alternative to conventional treatment. It is provided through an online platform, with the content tailored according to different types of interactions and algorithms [12]. dCBTi is available in a number of versions. One version func- tions as ancillary therapy to enhance the treatment effects of existing face-to-face $\mathrm{CBTi}$, another version provides feedback from a therapist online, and yet another version provides feedback based on a fully automated algorithm [13]. Advantages of dCBTi include immediate support, flexible scheduling, personalized content, and non-face-to-face interactions. The ability to visually present treatment progress or review past data is another advantage of dCBTi [13]. However, dCBTi relies entirely on the user's motivation and involves self-training that is performed alone without a therapist or other participants, which can lead to lack of adherence and ultimate treatment failure. Over-generalized advice, technical problems, and privacy or confidentiality issues are other major drawbacks [12]. In addition, most published randomized clinical trials have focused on a specific race, people living in a specific continent, or middle-aged individuals. Very few randomized studies have been conducted in Asia, a continent with high smartphone and internet usage [10]. Further studies involving populations of different cultures, ethnicities, and ages are required. Additionally, many studies have been conducted in patients with only mild to moderate insomnia and have not reported longer-term results after the dCBTi study period (typically 4-24 weeks) $[14,15]$. The effects of dCBTi compared with conventional CBTi are also unclear, as most studies compared dCBTi with wait-list or sham controls. Of the very limited number of randomized clinical studies comparing dCBTi with face-to-face CBTi, one showed similar results between methods, whereas the other reported inferior results with dCBTi. Of note, the study with similar results demonstrated non-inferiority for only the primary outcome, with dCBTi being inferior for secondary outcome variables, including adverse events, remission, response rate, depression, and use of sleep medication. The increase in medical claims related to DTx prescriptions should also be considered. As with prescriptions for drugs or other health care, prescribing DTx will increase overall health care costs. However, the effects on the opportunity cost of accessing other health care are unclear and will likely be controversial.

Several DTx for insomnia are currently on the market. Somryst $^{\mathbb{B}}$ (Pear Therapeutics), an FDA-approved DTx for insomnia, is a mobile application based on CBTi, which consists of 6 core sessions for a total of 9 weeks of therapy [16]. Treatment effects of Somryst ${ }^{\mathbb{Q}}$ were demonstrated in a randomized controlled trial that showed significant improvement of insomnia severity index (ISI) and sleep indicators, including sleep onset latency (SOL) and wake after sleep onset (WASO), compared with a patient education group [17]. Sleepio ${ }^{\mathrm{TM}}$ (Big Health Inc.) was developed jointly in the United Kingdom (UK) and US and is available as a computer program or mobile application. Sleepio ${ }^{\mathrm{TM}}$ is marked by Conformité Européenne (CE), indicating that it fulfilled safety, healthy, and environmental protection requirements of the European Union (EU) [18]. It consists of 6 core sessions for a total of 6 weeks of therapy. The therapeutic effects of Sleepio ${ }^{\mathrm{TM}}$ were verified in a randomized controlled trial showing significant improvements in sleep indicators, including sleep efficiency and daytime func- 
tioning, compared with imagery-relief therapy (placebo) or treatment as usual [19]. Sleepio ${ }^{\text {TM }}$ is now actively used in the health care system in the UK. Somnio ${ }^{\mathrm{TM}}$ (Mementor DE GmbH) is a mobile application consisting of 6 core sessions over a 6 -week period, which was approved as a German prescription digital health application (Digitale Gesundheitsanwendungen [DiGA]) in 2019 [20]. Its effectiveness was demonstrated in a randomized controlled trial reporting improvements in sleep indicators, including SOL, WASO, and daytime performance [21]. Although not approved as DTx by regulatory authorities, several other DTx for insomnia are also on the market, including BetterNight Insomnia $^{\mathrm{TM}}$ (BetterNight, LLC), a smartphone application shown to improve sleep deprivation symptoms and daytime somnolence [22], and the Night Owl - Sleep Coach ${ }^{\mathrm{TM}}$ (Mindware Consulting Inc.), a smartphone application demonstrated to improve sleep indicators after 2 months of treatment [23]. Sleep Ninja ${ }^{\text {TM }}$ (Black Dog Institute), a DTx for insomnia targeting adolescents, has also been shown to significantly improve sleep indicators [24].

Developing DTx for other sleep disorders, including obstructive sleep apnea (OSA) and narcolepsy, could be more difficult than developing DTx for insomnia because treatment of other sleep disorders usually requires more than just cognitive and behavioral changes. Most current digital health products targeting sleep disorders other than insomnia focus on evaluating or monitoring symptoms, with DTx functioning as a digital companion. No DTx for sleep disorders other than insomnia are being marketed at the current time. Mobile applications, wearable devices, and oxygen saturation monitors have been developed to evaluate and manage symptoms of OSA, including a smartphone application using a low-cost pulse oximeter, successful hypopnea monitoring using a finger pulse oximeter, and a smartphone application that monitors sleep apnea symptoms using a built-in sensor [25-28]. For narcolepsy, mobile applications monitoring changes in narcolepsy symptoms are being developed, which have resulted in increased self-efficacy in patients with narcolepsy $[29,30]$. For circadian sleep disorder, DTx could be used to restore and stabilize normal circadian rhythms by adjusting behavioral patterns, yet investigations in this field are still in the early stages [31]. Most DTx for sleep disorders other than insomnia have not received the approval of authorities as medical devices. Further studies verifying the therapeutic benefits of digital health products are required.

\section{USE OF DIGITAL THERAPEUTICS FOR SLEEP WITH PSYCHIATRIC COMORBIDITY}

CBTi has demonstrated effectiveness in reducing insomnia symptoms in patients with psychiatric comorbidity [32], and these benefits persist with dCBTi. Effectiveness of dCBTi has been reported for depression, anxiety disorder, and substance use disorder $[33,34]$. Studies have also shown that CBTi improves comorbid psychiatric symptoms, including depression and anxiety
$[32,35,36]$, and dCBTi has likewise been demonstrated to improve comorbid depression and anxiety symptoms [33,37]. Use of Somryst $^{\circledR}$ significantly improved depression symptoms evaluated with the Patient Health Questionnaire-9 (PHQ-9), with benefits persisting for 6 months after treatment in patients with comorbid depression and insomnia [38]. In a study of Sleepio ${ }^{\mathrm{TM}}, 68 \%$ of people with clinically significant elevated pre-treatment PHQ-9 or Generalized Anxiety Disorder-7 (GAD-7) scores had PHQ-9 or GAD-7 scores within the normal range after treatment [39]. Sleepio $^{\mathrm{TM}}$ has also been reported to significantly decrease Beck Anxiety Inventory (BAI) scores [40]. Another randomized controlled study demonstrated that Sleepio ${ }^{\mathrm{TM}}$ significantly reduced the ISI and Quick Inventory of Depression Symptomology [41]. Furthermore, Sleepio ${ }^{\mathrm{TM}}$ has been shown to improve quality of life [42] and cognitive function [43]. Improvements in PHQ-adolescent version and GAD-7 were also reported in a pilot study using Sleep Ninja ${ }^{\mathrm{TM}}$ [24].

\section{GLOBAL REGULATORY PROCESSES FOR DIGITAL THERAPEUTICS}

Discussions are ongoing regarding the establishment of standards for DTx regulation. DTA has stated that DTx used to treat or manage disease require third-party validation of effectiveness and safety claims by a regulatory or equivalent national body [4]. Since DTx have the characteristics of both SaMD and therapeutic agents, it is difficult to use existing regulatory methods for either medical devices or drugs. Several considerations exist regarding the regulation of DTx. Because DTx are in the form of software, and verifying practical functional methods, rather than operation methods, of these devices is necessary [4]. Also, because of the rapidly developing nature of digital technology, DTx products could possibly be outdated if the regulatory review period is prolonged. However, an excessively simplified regulatory process may result in insufficient clinical evidence [44]. In addition, because DTx are software, they require continuous updates and maintenance, which may lead to multiple regulatory review processes. Because DTx handle personal information of patients, cybersecurity must also be considered [45]. Lastly, although most DTx provide non-invasive treatment, and the possibility of toxicity or side effects is likely lower than that for other medical devices or drugs, confirming that there are no unexpected toxicities or side effects when DTx are used as therapeutic modalities is critical [5].

Regulatory methods for DTx are being established and developed worldwide. In the US, DTx have been granted approval or cleared by the FDA following submission of superiority trial data via either the de novo or $510(\mathrm{k})$ pathways, which are traditional regulatory process for medical devices [45]. The first FDA-approved DTx, reSET ${ }^{\circledR}$ (for substance use disorder), was certified via the de novo pathway, but the FDA subsequently recognized the limitations of regulating DTx using traditional regulatory processes for medical devices. It established a guidance for the clinical evaluation of SaMDs in 2017 [46], and a precertification pro- 
gram for new approval of DTx in 2018 [47]. This guidance considered DTx as having a higher risk of adverse effects than other SaMDs because of its therapeutic purpose and thus required DTx effectiveness and safety to be verified by independent clinical evidence. The pre-certification program for DTx was established to address concerns that prolonged regulatory processes would slow the speed of developing DTx and that regulation of maintenance and updates following product launch would be required. The core concepts of the precertification program are evaluation of the development process and manufacturing company, rather than evaluation of each program, and replacement of clinical evidence from clinical trials by real-world evidence, which can be obtained through real-life user experience. In the pre-certification program, which began as a pilot program in 2019 involving 9 companies, the overall authentication process involves evaluation of 5 core items: product quality, patient safety, clinical responsibility, cybersecurity responsibility, and proactive culture [47]. Somryst ${ }^{\mathbb{B}}$ was approved by the FDA via both the $510(\mathrm{k})$ pathway and precertification program.

The EU strengthened its SaMD regulations in 2017 by including SaMDs as a medical device in the new Medical Devices Regulation (MDR) released that year [48]. According to this new MDR, some software previously considered "low risk" could be up-classified, requiring notified body approval [49]. SaMDs were also required to receive CE marking, similar to other medical devices [50]. Overall, the regulatory processes for SaMDs were tightened in the revised MDR. Because of concerns that this revision could represent an overly burdensome regulatory process for rapidly changing digital health, the National Health Service-X (NHS$\mathrm{X}$ ) unit was created in the UK in 2019 to guide best practices for NHS technology, digital information, and data, including data sharing and transparency [51]. Under the leadership of NHS-X, all digital health products in the UK were classified according to the potential risk to patients, and guidelines were provided for minimum evidence requirements [52]. If a digital product is selected as a target by policy priority, the manufacturer could obtain support via an accelerated access pathway, allowing the product to be approved quickly [53]. In 2019, the Digital Healthcare Act was established in Germany, after which fast-track approval for digital health applications (DiGA) began [54]. By 2021, a total of 11 products were approved DiGA, including Somnio ${ }^{\mathrm{TM}}$.

In Korea, regulatory processes have been established for rapid and appropriate DTx regulation. In 2019, the Ministry of Food and Drug Safety (MFDS) announced guidelines for SaMD regulation and approval to improve the safety of the SaMD development and related contents [55]. The next year, the Medical Device Industry Promotion and Innovative Medical Device Support Act was announced by MFDS. With this act, some data required for approval could be exempted if the manufacturer submitted proper certification, similar to the precertification program in the US [56].

Discussions are ongoing to establish guidelines for the prescription and billing of DTx. DTA guidelines recommend that DTx designed for treating diseases be used with a doctor's prescription [4].
Use of Somryst ${ }^{\circledR}$, a DTx approved by the US FDA, requires a physician's prescription [16]. However, a clear standard has not yet been established with regard to the billing for DTx in the US. If the product is recognized as sufficiently valid and necessary, a review of insurance coverage will occur, which is the same process used for existing medical devices. To qualify for insurance reimbursement, manufacturers should submit an application with documents indicating the target population, purpose of use, and instructions for use [57]. In the UK, it is recommended that medical devices used for treatment purposes be prescribed by a physician [52]; however, Sleepio ${ }^{\mathrm{TM}}$ can be used without prescription by focusing on improvements in life style, including sleep [18]. Billing for DTx use is determined by collecting and evaluating data after product review in the UK [58]. In Germany, DTx approved via DiGA can be prescribed and billed for. For the first year after release of a product, the manufacturers can set the medical fee; thereafter, the fee may be changed by negotiation with German health insurance [54,59]. In Korea, no guidelines exist regarding the prescription or billing of DTx. As with existing medical devices, all decisions about how to charge and pay for DTx should consider various factors, including the opinions of experts, academic opinions, medical feasibility, clinical significance and therapeutic effectiveness, cost effectiveness, cost burden to the patient, and social benefits $[60,61]$.

\section{CHALLENGES OF DIGITAL THERAPEUTICS}

The main target of DTx is chronic disorders, including insomnia. This is because health-risk behaviors and poor adherence to medical regimens can have major effects on the development and management of chronic disease. Self-regulation (the ability to manage cognitive, motivational, and emotional resources that enable individuals to act according to their long-term goals) is an important determinant of health behavior and outcomes. As self-regulation is a major subject of interventions for chronic disease, engagement in the use of DTx is a very important consideration. Although the abovementioned dCBTi is effective not only for insomnia symptoms but also for psychiatric symptoms, such as depression and anxiety, these results were obtained under specific experimental conditions and may not reflect real-world situations. It is generally known that use of DTx in real-world settings is decreasing, and engagement with DTx remains the most important issue to address [62].

Studies on the treatment of depression and anxiety with DTx found that participants' adherence to internet-provided cognitive behavioral therapy varied from $6 \%$ to $100 \%$ [63]. Similarly, reviews of DTx as a digital companion found that engagement varies widely $[62,64]$ and may be lower than that of traditional treatments [65]. User engagement refers to the user's understanding and ongoing interaction with digital interventions. User engagement includes expression of interest (such as registering for DTx), active use of DTx (such as participation in research), and contin- 
ued use of DTx, even after the minimum period required for research participation has elapsed [66]. User factors that can influence engagement include demographic characteristics, personality, psychiatric comorbidity, attitude toward mobile application use, and familiarity with mobile devices. Program factors that may influence engagement include the quality of the content provided through DTx, method of delivery, level of recommendations, and presence of peer support. Technical factors include confidentiality and privacy issues, as well as how accurately and easily the program functions.

\section{SUMMARY}

As a software-based therapeutic intervention, DTx represent a new treatment paradigm. DTx provide opportunities for healthcare professionals to offer digitalized, personalized medicine to help patients manage their own diseases. This characteristic of DTx as a form of personalized medicine could be especially important in the field of sleep medicine because sleep disorders are typically chronic diseases closely related to a person's lifestyle. Today, the most well developed and widely used DTx in the sleep field are DTx for insomnia. Considering the possible side effects of long-term use of hypnotics and the low accessibility of traditional CBTi, DTx for insomnia could be a particularly useful approach for treating chronic insomnia.

However, a number of issues remain unclear regarding the clinical use of DTx in the field of sleep medicine. Firstly, DTx for sleep disorders other than insomnia have not yet been sufficiently developed. Further research is necessary to provide sufficient clinical evidence of the effectiveness and safety of the treatment effects of DTx for these other disorders. Secondly, issues related to regulation of DTx have not yet been clarified. Each country or region has established a system for regulation, prescription, and medical billing of DTx, but these systems are still in the early stages. Lastly, user engagement must be considered a core feature when developing DTx. Because of their focus on cognitive and behavioral modifications, DTx typically require more time and effort than pharmacotherapeutics, which may limit their use in the real world if user engagement is inadequate. Nevertheless, with continued interest and efforts of healthcare professionals in the field of sleep medicine to address the issues related to DTx, these technologies could become widely used as an effective therapeutic option for sleep disorders in the future.

\section{Acknowledgments}

The National Research Foundation of Korea funded by the Ministry of Science, ICT \& Future Planning, Republic of Korea supported the present work (Grant number: 2017R1A2B3008214 to Eun Lee). The funding source had no involvement in the study design, data collection, or writing of the report.

\section{Conflicts of Interest}

The authors have no potential conflicts of interest to disclose.

\section{Author Contributions}

Conceptualization: all authors. Data curation: all authors. Funding acquisition: Eun Lee. Investigation: Kyung Mee Park, Suonaa Lee. Methodology: Kyung Mee Park, Eun Lee. Project administration: Eun Lee. Resources: all authors. Supervision: Kyung Mee Park, Eun Lee. Validation: Eun Lee. Writing_original draft: Kyung Mee Park, Eun Lee. Writing_review \& editing: Kyung Mee Park, Eun Lee.

\section{ORCID iDs}

Kyung Mee Park

https://orcid.org/0000-0002-2416-2683

Suonaa Lee (1)

https://orcid.org/0000-0003-1801-3267

Eun Lee (1)

https://orcid.org/0000-0002-7462-0144

\section{REFERENCES}

1. Sepah SC, Jiang L, Peters AL. Long-term outcomes of a Web-based diabetes prevention program: 2-year results of a single-arm longitudinal study. J Med Internet Res 2015;17:e92.

2. Pear Therapeutics. reSET ${ }^{\circledR} \&$ reSET-O ${ }^{\circledR}$ [Internet]. Available at: https:// peartherapeutics.com/products/reset-reset-o/. Accessed October 17, 2021.

3. Welldoc. Bluestar 2010 [Internet]. Available at: https://www.welldoc.com/. Accessed October 17, 2021.

4. Digital Therapeutics Alliance. Digital therapeutics definition and core principles [Internet]. Available at: https://dtxalliance.org/understanding-dtx/. Accessed October 17, 2021.

5. Dang A, Arora D, Rane P. Role of digital therapeutics and the changing future of healthcare. J Family Med Prim Care 2020;9:2207-2213.

6. Hong JS, Wasden C, Han DH. Introduction of digital therapeutics. Comput Methods Programs Biomed 2021;209:106319.

7. Joyce M, Leclerc O, Westhues K, Xue H. Digital therapeutics: preparing for takeoff. New York: McKinsey \& Company; 2018.

8. Riemann D, Baglioni C, Bassetti C, Bjorvatn B, Dolenc Groselj L, Ellis JG, et al. European guideline for the diagnosis and treatment of insomnia. J Sleep Res 2017;26:675-700.

9. Morin CM, Vallières A, Guay B, Ivers H, Savard J, Mérette C, et al. Cognitive behavioral therapy, singly and combined with medication, for persistent insomnia: a randomized controlled trial. JAMA 2009;301:2005-2015.

10. Lee E. Cognitive behavioral treatment as a digital therapeutic for insomnia. Chronobiol Med 2020;2:10-15.

11. Weyerer S, Dilling H. Prevalence and treatment of insomnia in the community: results from the upper Bavarian field study. Sleep 1991;14:392-398.

12. Espie CA, Hames P, McKinstry B. Use of the internet and mobile media for delivery of cognitive behavioral insomnia therapy. Sleep Med Clin 2013; 8:407-419.

13. Luik AI, Kyle SD, Espie CA. Digital cognitive behavioral therapy (dCBT) for insomnia: a state-of-the-science review. Curr Sleep Med Rep 2017;3:48-56.

14. Blom K, Tarkian Tillgren H, Wiklund T, Danlycke E, Forssén M, Söderström A, et al. Internet- vs. group-delivered cognitive behavior therapy for insomnia: a randomized controlled non-inferiority trial. Behav Res Ther 2015;70:47-55

15. Lancee J, van Straten A, Morina N, Kaldo V, Kamphuis JH. Guided online or face-to-face cognitive behavioral treatment for insomnia: a randomized wait-list controlled trial. Sleep 2016;39:183-191.

16. Pear Therapeutics. Somryst ${ }^{\mathbb{B}}$ [Internet]. Available at: https://peartherapeutics.com/products/somryst/. Accessed October 24, 2021.

17. Ritterband LM, Thorndike FP, Ingersoll KS, Lord HR, Gonder-Frederick L, Frederick C, et al. Effect of a web-based cognitive behavior therapy for insomnia intervention with 1-year follow-up: a randomized clinical trial. JAMA Psychiatry 2017;74:68-75.

18. Big Health. Sleepio [Internet]. Available at: https://www.sleepio.com/. Ac- 
cessed October 24, 2021.

19. Espie CA, Kyle SD, Williams C, Ong JC, Douglas NJ, Hames P, et al. A randomized, placebo-controlled trial of online cognitive behavioral therapy for chronic insomnia disorder delivered via an automated media-rich web application. Sleep 2012;35:769-781.

20. Mementor. Somnio [Internet]. Available at: https://somn.io/en/. Accessed November 4, 2021.

21. Lorenz N, Heim E, Roetger A, Birrer E, Maercker A. Randomized controlled trial to test the efficacy of an unguided online intervention with automated feedback for the treatment of insomnia. Behav Cogn Psychother 2019;47: 287-302.

22. Munafo D, Loewy D, Reuben K, Kavy G, Hevener B. Sleep deprivation and the workplace: prevalence, impact, and solutions. Am J Health Promot 2018:32:1644-1646.

23. Harbison BR, Cole J, Wetzler R, Gorzynski A. 0408. Evaluation of a CBT-I self-help program administered by a mobile app. Sleep 2018;41:A155.

24. Werner-Seidler A, Wong Q, Johnston L, O’Dea B, Torok M, Christensen H. Pilot evaluation of the Sleep Ninja: a smartphone application for adolescent insomnia symptoms. BMJ Open 2019;9:e026502.

25. Zhang Z, Qi M, Hügli G, Khatami R. The challenges and pitfalls of detecting sleep hypopnea using a wearable optical sensor: comparative study. J Med Internet Res 2021;23:e24171

26. Penzel T, Schöbel C, Fietze I. New technology to assess sleep apnea: wearables, smartphones, and accessories. F1000Res 2018;7:413.

27. Alqassim S, Ganesh M, Khoja S, Zaidi M, Aloul F, Sagahyroon A. Sleep apnea monitoring using mobile phones. Proceedings of the 2012 IEEE 14th International Conference on e-Health Networking, Applications and Services (Healthcom); 2012 Oct 10-13; Beijing, China: IEEE; 2012, p. 443-446.

28. Petersen CL, Chen TP, Ansermino JM, Dumont GA. Design and evaluation of a low-cost smartphone pulse oximeter. Sensors (Basel) 2013;13:1688216893.

29. Staszak W, de Paula D, Uebernickel F. The power of habits: evaluation of a mobile health solution for the management of narcolepsy. Proc Design Soc 2021;1:3081-3090.

30. Quaedackers L, De Wit J, Pillen S, Van Gilst M, Batalas N, Lammers GJ, et al. A mobile app for longterm monitoring of narcolepsy symptoms: design, development, and evaluation. JMIR Mhealth Uhealth 2020;8:e14939.

31. Cho CH, Lee HJ. Applying circadian rhythm concepts in digital healthcare. Chronobiol Med 2021;3:1-3.

32. Taylor DJ, Pruiksma KE. Cognitive and behavioural therapy for insomnia (CBT-I) in psychiatric populations: a systematic review. Int Rev Psychiatry 2014;26:205-213.

33. Ye YY, Zhang YF, Chen J, Liu J, Li XJ, Liu YZ, et al. Internet-based cognitive behavioral therapy for insomnia (ICBT-i) improves comorbid anxiety and depression - a meta-analysis of randomized controlled trials. PLoS One 2015;10:e0142258.

34. Hermes ED, Rosenheck RA. Implementing computer-based psychotherapy among veterans in outpatient treatment for substance use disorders. Psychiatr Serv 2016;67:176-183.

35. Belleville G, Cousineau H, Levrier K, St-Pierre-Delorme MÈ. Meta-analytic review of the impact of cognitive-behavior therapy for insomnia on concomitant anxiety. Clin Psychol Rev 2011;31:638-652.

36. Cunningham JEA, Shapiro CM. Cognitive behavioural therapy for insomnia (CBT-I) to treat depression: a systematic review. J Psychosom Res 2018; 106:1-12.

37. Thorndike FP, Ritterband LM, Gonder-Frederick LA, Lord HR, Ingersoll KS, Morin CM. A randomized controlled trial of an internet intervention for adults with insomnia: effects on comorbid psychological and fatigue symptoms. J Clin Psychol 2013;69:1078-1093.

38. Christensen H, Batterham PJ, Gosling JA, Ritterband LM, Griffiths KM, Thorndike FP, et al. Effectiveness of an online insomnia program (SHUTi) for prevention of depressive episodes (the GoodNight Study): a randomised controlled trial. Lancet Psychiatry 2016;3:333-341.

39. Luik AI, Bostock S, Chisnall L, Kyle SD, Lidbetter N, Baldwin N, et al. Treating depression and anxiety with digital cognitive behavioural therapy for insomnia: a real world NHS evaluation using standardized outcome measures. Behav Cogn Psychother 2017;45:91-96.

40. Pillai V, Anderson JR, Cheng P, Bazan L, Bostock S, Espie CA, et al. The anxiolytic effects of cognitive behavior therapy for insomnia: preliminary results from a web-delivered protocol. J Sleep Med Disord 2015;2:1017.

41. Cheng P, Luik AI, Fellman-Couture C, Peterson E, Joseph CLM, Tallent G, et al. Efficacy of digital CBT for insomnia to reduce depression across demographic groups: a randomized trial. Psychol Med 2019;49:491-500.

42. Espie CA, Emsley R, Kyle SD, Gordon C, Drake CL, Siriwardena AN, et al. Effect of digital cognitive behavioral therapy for insomnia on health, psychological well-being, and sleep-related quality of life: a randomized clinical trial. JAMA Psychiatry 2019;76:21-30.

43. Kyle SD, Hurry MED, Emsley R, Marsden A, Omlin X, Juss A, et al. The effects of digital cognitive behavioral therapy for insomnia on cognitive function: a randomized controlled trial. Sleep 2020;43:zsaa034.

44. Doss BD, Weingardt KR, Lindhiem OJ, Timmons AC, Jones DJ, Comer JS, et al. Issues in regulating DTx [Internet]. Available at: https://www.psychiatrictimes.com/view/issues-in-regulating-dtx. Accessed November 14, 2021.

45. Patel NA, Butte AJ. Characteristics and challenges of the clinical pipeline of digital therapeutics. NPJ Digit Med 2020;3:159.

46. FDA. Software as a medical device (SAMD): clinical evaluation [Internet]. Available at: https://www.fda.gov/regulatory-information/search-fda-guidance-documents/software-medical-device-samd-clinical-evaluation. Accessed October 30, 2021.

47. FDA. Developing a software precertification program: a working model [Internet]. Available at: https://www.fdanews.com/ext/resources/files/2018/0621-18-Precertification.pdf?1529601502. Accessed November 3, 2021.

48. European Union. Regulation (EU) 2017/746 of the European Parliament and of the Council of 5 April 2017 on in vitro diagnostic medical devices and repealing Directive 98/79/EC and Commission Decision 2010/227/EU [Internet]. Available at: https://eur-lex.europa.eu/legal-content/EN/TXT/HT $\mathrm{ML} /$ ? uri $=$ CELEX:32017R0746\&from $=\mathrm{EN} \% 23 \mathrm{~d} 1 \mathrm{e} 1005-176$ 1\#d1e1129-176-1. Accessed November 3, 2021.

49. European Union. Regulation (EU) 2017/745 of the European Parliament and of the Council of 5 April 2017 on medical devices, amending directive 2001/83/EC, Regulation (EC) no 178/2002 and Regulation (EC) no 1223/2009 and repealing Council Directives 90/385/EEC and 93/42/EEC (text with EEA relevance) [Internet]. Available at: https://eur-lex.europa.eu/legal-content/ EN/TXT/?uri=CELEX:32017R0745. Accessed November 3, 2021.

50. Boehringer Ingelheim. An innovator's guide to the NHS: navigating the barriers to digital health [Internet]. Available at: https://www.boehringer-ingelheim.co.uk/sites/gb/files/documents/innovators_guide.pdf. Accessed November 3, 2021.

51. National Health Service. NHSx [Internet]. Available at: https://www.nhsx. nhs.uk/. Accessed November 3, 2021.

52. NICE. Evidence standards framework for digital health technologies [Internet]. Available at: https://www.nice.org.uk/about/what-we-do/our-programmes/evidence-standards-framework-for-digital-health-technologies. Accessed November 13, 2021.

53. GOV.UK. Accelerated access review: final report [Internet]. Available at: https://www.gov.uk/government/publications/accelerated-access-review-final-report. Accessed November 13, 2021.

54. Federal Institute for Drugs and Medical Devices. The fast-track process for digital health applications (DiGA) according to section 139e SGB V [Internet]. Available at: https://www.bfarm.de/SharedDocs/Downloads/EN/ MedicalDevices/DiGA_Guide.html. Accessed November 13, 2021.

55. Ministry of Food and Drug Safety. Guideline for software as a medical device approval [Internet]. Available at: http://www.nifds.go.kr/brd/m_15/ view.do?seq=12715. Accessed November 3, 2021.

56. Ministry of Food and Drug Safety. Medical Device Industry Promotion and Innovative Medical Device Support Act 2020 [Internet]. Available at: https:// www.law.go.kr/법령/의료기기산업육성및혁신의료기기지원법. Accessed November 3, 2021.

57. U.S. Centers for Medicare \& Medicaid Services. Medicare coverage determination process [Internet]. Available at: https://www.cms.gov/Medicare/ Coverage/DeterminationProcess. Accessed November 13, 2021.

58. National Health Service. Adult improving access to psychological therapies programme [Internet]. Available at: https://www.england.nhs.uk/mentalhealth/adults/iapt/. Accessed November 13, 2021.

59. Shin J. What we have to prepare for being smart in healthcare: based on the Korean current situation and long-term direction of the national health insurance. HIRA Policy Brief 2021;15:20-28. 
60. National Evidence-based Healthcare Collaborating Agency. Easy-to-understand new medical technology assessment system A to Z [Internet]. Available at: https://nhta.neca.re.kr/nhta/publication/nhtaU0605V. ecg?seq=8975. Accessed November 13, 2021.

61. Health Insurance Review \& Assessment Service. Guidelines for evaluation of medical care benefits in innovative medical technology [Internet]. Available at: http://www.hira.or.kr/bbsDummy.do?pgmid=HIRAA02000200010 0\&brdScnBltNo=4\&brdBltNo=7655. Accessed November 13, 2021.

62. Baumel A, Edan S, Kane JM. Is there a trial bias impacting user engagement with unguided e-mental health interventions? A systematic comparison of published reports and real-world usage of the same programs. Transl Behav Med 2019;9:1020-1033.

63. Andrews G, Basu A, Cuijpers P, Craske MG, McEvoy P, English CL, et al.
Computer therapy for the anxiety and depression disorders is effective, acceptable and practical health care: an updated meta-analysis. J Anxiety Disord 2018;55:70-78.

64. Fleming T, Bavin L, Lucassen M, Stasiak K, Hopkins S, Merry S. Beyond the trial: systematic review of real-world uptake and engagement with digital self-help interventions for depression, low mood, or anxiety. J Med Internet Res 2018;20:e199.

65. Kaltenthaler E, Sutcliffe P, Parry G, Beverley C, Rees A, Ferriter M. The acceptability to patients of computerized cognitive behaviour therapy for depression: a systematic review. Psychol Med 2008;38:1521-1530.

66. Borghouts J, Eikey E, Mark G, De Leon C, Schueller SM, Schneider M, et al. Barriers to and facilitators of user engagement with digital mental health interventions: systematic review. J Med Internet Res 2021;23:e24387. 